Published in "Ecology 83(9): 2394-2407, 2002"

which should be cited to refer to this work.

\title{
QUANTITATIVE DESCRIPTORS OF FOOD-WEB MATRICES
}

\author{
Louis-Félix Bersier, ${ }^{1,2,3}$ CARolin Banašek-Richter, ${ }^{1}$ And MARIE-France CAtTin ${ }^{1}$ \\ ${ }^{1}$ Zoological Institute, University of Neuchâtel, Rue Emile-Argand 11, CH-2007 Neuchâtel, Switzerland \\ ${ }^{2}$ Department of Ecology, University of Fribourg, Pérolles, CH-1700 Fribourg, Switzerland
}

\begin{abstract}
A food web customarily describes the qualitative feeding relationships in a community. Descriptors have been used to extract ecologically meaningful information from such data, e.g., the proportion of top species (the proportion of taxa without consumers) or vulnerability (the average number of consumers per taxon). Analyses of collections of food webs based on these properties have revealed regularities that fostered the formulation of models of food-web structure. However, it has been shown that most of these qualitative descriptors are highly sensitive to the varying levels of sampling effort used to document a food web. The principal problem is that webs described extensively include trophic links of highly uneven magnitude, with typically few strong/important links and a wealth of weak ones; with qualitative descriptors, the same weight is given to all trophic interactions. To overcome this problem, food webs should be described and analyzed quantitatively. Consequently, we propose here a suite of food-web descriptors, which are built on information-theory indices and take the magnitude of the trophic interactions into account. We define descriptors having a similar meaning as the classical qualitative indices. Two versions of each quantitative descriptor are proposed, one giving the same weight to each taxon, and one weighting each taxon by the total amount of its incoming and outgoing biomass flows. We use a published quantitative food web to exemplify the computation of the new descriptors, and discuss their potential and limitations.
\end{abstract}

Key words: Chesapeake Bay (USA) mesohaline ecosystem; connectance; food web, quantitative descriptors; information-theory indices; omnivory; sampling effect; Shannon index; trophic links.

\section{INTRODUCTION}

A community food web describes the feeding relationships among the taxa comprising a community. Analyses of initial collections of community food webs have identified a suite of intriguing regularities; some web properties (among others, the fractions of top, intermediate, and basal taxa, the number of links per taxa, rigid circuits) were found to be scale invariant-they stay roughly constant across a variety of webs spanning a wide range in the number of taxa they contain (e.g., Cohen 1977, 1989, Pimm 1982, Briand and Cohen 1984, Lawton 1989, Sugihara et al. 1989, Pimm et al. 1991, Havens 1992). These findings fostered the formulation of models explaining the structure of these biological networks (Sugihara 1984, Cohen and Newman 1985). However, the quality of the data set, which had been gathered from the literature, was rightfully criticized (e.g., May 1983, Paine 1988). Subsequently

\footnotetext{
${ }^{3}$ E-mail: Louis-Felix.Bersier@unine.ch
}

compiled food webs, aimed specifically at documenting the feeding relationships in communities, did not uphold the initially found regularities (Warren 1989, Winemiller 1990, Martinez 1991, Polis 1991, Deb 1995, Carney et al. 1997), and new hypotheses about the structure of communities have been proposed (Martinez 1992, Polis and Strong 1996, Solow and Beet 1998, Williams and Martinez 2000). In all these studies, food webs have been analyzed with qualitative descriptors, and it has been shown that most of these indices are extremely sensitive to different levels of sampling effort (Goldwasser and Roughgarden 1997, Martinez et al. 1999). In the same vein, Bersier et al. (1999) showed that the scaling behavior of the link-density property was affected by varying levels of sampling effort; using two models and empirical data, they found that low sampling effort tends to produce the appearance of scale invariance in intrinsically scale-dependent systems.

These sampling effects highlight the following problem inherent to qualitative food webs. The distribution of link importance in highly resolved food webs is likely to be strongly uneven (see e.g., Goldwasser and 
Roughgarden 1993). In qualitative food webs, the same weight is given to all links, which distorts the true picture of the structure of the food webs (Kenny and Loehle 1991). This points to the need for quantitative data on link importance, which allows a more sensible approach to food-web structure (e.g., May 1983, Kenny and Loehle 1991, Pimm et al. 1991, Cohen et al. 1993, Bersier et al. 1999). To make use of such data, we must think of alternative ways of defining food-web properties that take the disequitability in the distribution of link importance into account. Quantitative descriptors have already been proposed, but generally with aims that differ from those of classical food-web properties. For example, ascendancy (Ulanowicz 1986) and mutual information (Hirata 1995) are used as macro-descriptors of complex systems; they are typically applied to large ecosystems, whose compartments are highly aggregated, and aim at expressing the phenomenology of growth and development of these systems (Ulanowicz 1997).

In this paper we propose a suite of descriptors that can be applied to quantitative food webs. We chose to derive our descriptors from information theory, and tried to propose indices having a similar meaning as those customarily used to describe qualitative food webs. The computation of each property is exemplified by the use of a published quantitative food web, the Chesapeake Bay (east coast, USA) mesohaline ecosystem (Baird and Ulanowicz 1989).

\section{The Food-Web Matrix}

A qualitative food web composed of $s$ taxa, can be represented by an $s$-by-s predation matrix $\mathbf{a}=\left[a_{i j}\right]$, with

$$
a_{i j}= \begin{cases}1 & \text { if taxa } j \text { preys on taxa } i \\ 0 & \text { otherwise }\end{cases}
$$

and $a_{i}$. the sum of row $i, a_{\text {.j }}$ the sum of column $j$, and $a_{\text {.. }}$ the sum of a. This matrix can be represented graphically as a connected directed graph whose vertices are the taxa, and the edges the trophic interactions (Fig. 1). In a quantitative food web, it is necessary to assign some unit to $a_{i j}$. The quantitative descriptors presented here are suitable for quantitative measures whose units are comparable row- and column-wise, for example for flow of carbon (e.g., milligrams of carbon per square meter per day). In the following, we will use the general term flow of biomass when referring to these quantitative measures. To avoid confusion, we will call such a matrix b, with elements $b_{i j}$ defined as

$$
\begin{gathered}
b_{i j}=\text { biomass passing from taxon } i \text { to taxon } j \\
\text { per unit surface area and time. }
\end{gathered}
$$

The Chesapeake Bay ecosystem (Baird and Ulanowicz 1989) depicted in the Appendix and Fig. 1 is an example of a quantitative food web. This data set was recently included in an analysis by Williams and Mar- tinez (2000) as one of the largest and highest-quality empirical food webs. However, lower taxa are still highly aggregated.

\section{Qualitative Descriptors}

Food-web matrices are complex objects, and descriptors have been devised to extract ecologically meaningful information from these entities. The qualitative descriptors for which we propose quantitative counterparts can be classified into different groups.

\section{Species properties}

In a food web, a taxon $(k)$ is top if it has prey (denoted by " $N$ ") but no consumers (denoted by " $\mathrm{P}$ "), intermediate if it has prey and consumers, and basal if it only has consumers. Four properties have been built based on these categories: the ratio of prey to consumers (N:P) and the proportion of top $(\% T)$, of intermediate $(\% I)$, and of basal species $(\% B)$. Note that these are not independent measures as $\% T+\% I+\% B$ $=1$, and $\mathrm{N}: \mathrm{P}=(\% B+\% I) /(\% I+\% T)$. The ratio of consumers to prey has been found to cluster about 1 in collections of food webs, which means that, on average, taxa have the same number of prey as of consumers. This pattern has been criticized as a mathematical inevitability in large webs (Closs et al. 1995), which all have a large proportion of intermediate taxa (Martinez and Lawton 1995).

\section{Link properties}

This category includes the link density (LD) and the various measures of connectance (see Warren [1994] for a list of definitions), which are all based on the total number of trophic links $(l)$ and on the number of taxa in food web $(s)$. They play a key role in community ecology because they lie at the heart of many theories of community stability (May 1974, Pimm 1984) and structure (Martinez 1992, Solow and Beet 1998). The link density is measured simply as $l / s$; a widely used measure of connectance is $l / s^{2}$, called directed connectance $(C)$, which corresponds to the number of actual links over the number of possible links, including cannibalistic loops (Martinez 1992). The proportions of links among the three categories of taxa also fall in this category, i.e., between top and intermediate (\% TI), top and basal (\%T-B), intermediate and intermediate $(\% \mathrm{I}-\mathrm{I})$, and intermediate and basal taxa (\%I-B). Again, these four proportions are not independent as their sum equals 1 .

\section{Chain properties}

A food chain is a distinct path within the food-web matrix from any taxon down to a basal taxon. A chain linking a top and a basal taxon is called a maximal food chain (mfc) (Fig. 1). Several measures using food chains capture the complexity of the food web: the number of maximal food chains $\left(n_{\mathrm{mfc}}\right)$, and several regarding chain length $(\mathrm{cl})$ : the mean $\left(M_{\mathrm{cl}}\right)$, median 


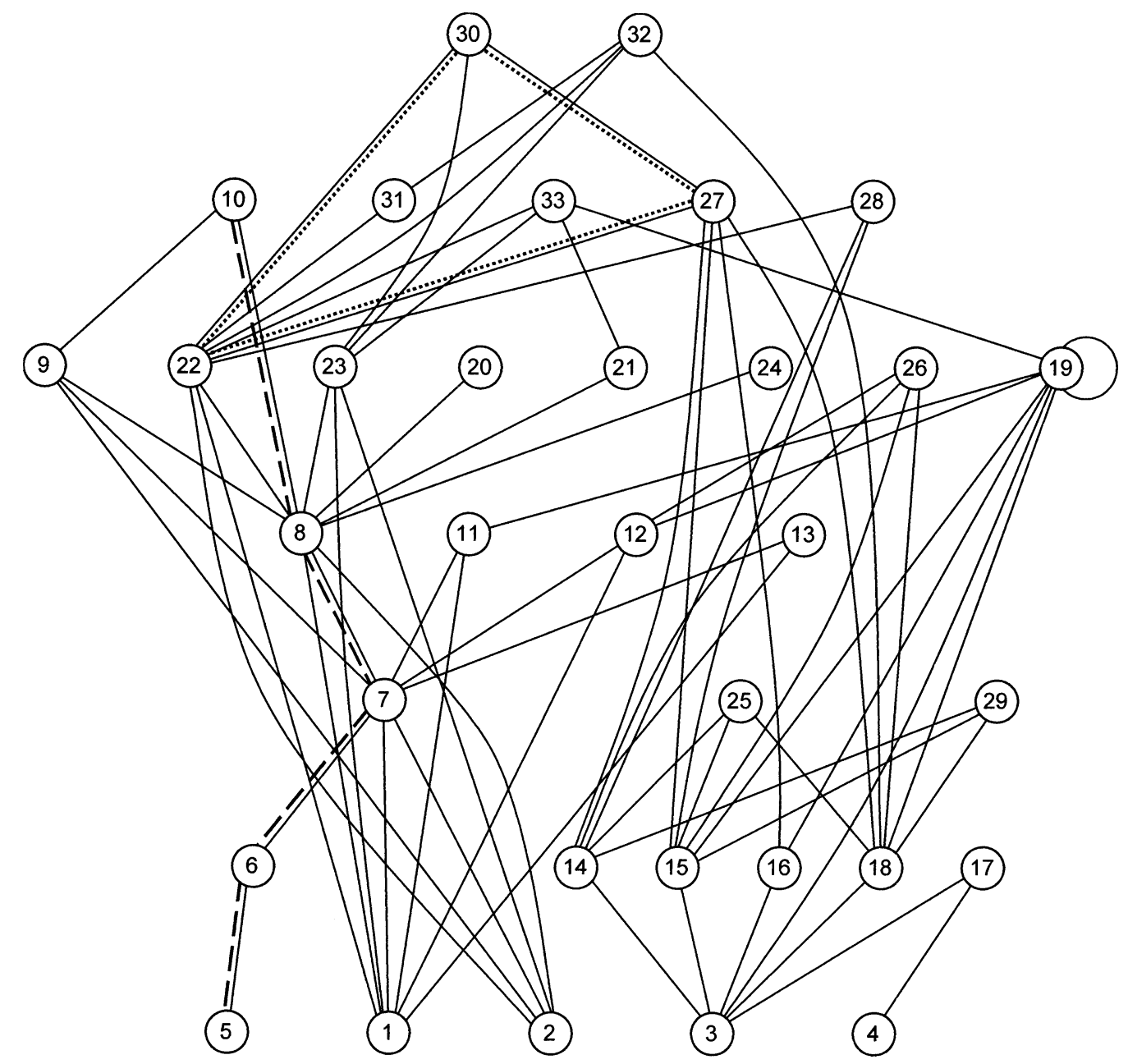

FIG. 1. Connected directed graph representing the trophic interactions among the 33 major taxa of the Chesapeake Bay mesohaline ecosystem (Baird and Ulanowicz 1989). Data and the list of taxa are in the Appendix. Taxa are arranged according to their trophic level (consumers are always above their prey). Dashed line = example of a maximal food chain; dotted line $=$ example of a closed omnivorous link.

$\left(\mathrm{MED}_{\mathrm{cl}}\right)$, standard deviation $\left(\mathrm{SD}_{\mathrm{cl}}\right)$, and maximum $\left(\mathrm{MAX}_{\mathrm{cl}}\right)$ of the lengths of maximal food chains. However, the presence of loops (e.g., A eats B and B eats A) can render the computation of properties based on maximal chain length problematic, because it is possible to have no top taxon. Therefore an approach that avoids this difficulty is to calculate chain properties including all food chains, rather than just maximal chains (e.g., Williams and Martinez 2000).

\section{Omnivory properties}

A taxon is omnivorous if it consumes prey belonging to different trophic levels. There are several ways of defining the trophic level of a taxon (Yodzis 1989); the characterization we adopt here is "one plus the length of the longest chain from the focal taxon to a basal taxon." The degree of omnivory can be measured simply as the proportion of taxa that consume prey from more than one trophic level $(\% O)$. At least two other measures of omnivory have been proposed in the literature. The first one is the number of closed omnivorous links divided by the number of top taxa (Sprules and Bowerman 1988, Hall and Raffaelli 1991). A closed omnivorous link is a loop starting from the consumer to a prey more than one trophic level away, and back to the consumer through at least one other prey located at an intermediate trophic level (see Fig. 1). The second one is the mean of the standard deviations in chain lengths over all taxa (Goldwasser and Roughgarden 1993).

\section{Consumer-prey asymmetries}

The vulnerability $(V)$ and generality $(G)$ properties were introduced by Schoener (1989) as the mean number of consumers per prey, and the mean number of prey per consumer, respectively. They can be computed 
easily as $l /\left(n_{\mathrm{B}}+n_{\mathrm{I}}\right)$, and $l /\left(n_{\mathrm{T}}+n_{\mathrm{I}}\right)$, respectively, with $n_{\mathrm{T}}$ the number of top, $n_{\mathrm{I}}$ the number of intermediate, and $n_{\mathrm{B}}$ the number of basal taxa. Williams and Martinez (2000) introduced a measure of the variability for $V$ and $G$, the standard deviation of normalized generality $(\operatorname{sd} G)$, and of normalized vulnerability $(\mathrm{sd} V)$. For taxon $k$, normalized $G_{k}$ and $V_{k}$ are

$$
\begin{aligned}
G_{k} & =\frac{1}{l / s} \sum_{i=1}^{s} a_{i k} \\
V_{k} & =\frac{1}{l / s} \sum_{j=1}^{s} a_{k j}
\end{aligned}
$$

which forces mean $G_{k}$ and $V_{k}$ to equal 1, and allows comparisons across webs of different sizes.

\section{Other descriptors}

Several other properties, from simple to elaborate in computations, have been proposed, e.g., the proportion of intermediate links by consumers only (Goldwasser and Roughgarden 1997), the mean maximum trophic similarity of each taxon in the food web (Williams and Martinez 2000), the lumpability of a food web (Solow and Beet 1998), as well as topological properties of graphical representations of food webs, i.e., intervality (Cohen 1978) and the rigid circuit property for niche overlap graphs, and the presence of topological holes for resource graphs (Sugihara 1982, 1984). Since these descriptors are not widely used, they will not be considered here.

\section{Quantitative Descriptors}

The catalyst for the formulation of the quantitative descriptors presented here is the work of Ulanowicz and Wolff (1991) on effective connectance. Like these authors, we use information-theoretical indices, particularly the Shannon (Shannon 1948) measure of entropy (or uncertainty), $H$. The reader can refer to Ulanowicz (1997) for a didactical introduction to the concept, or to Ulanowicz (1986) for a more technical treatment. For a given number $x$ of events, $H$ reaches its maximum when all events occur in equal proportion $(H=\log x)$; the minimum is a function of the number of cases in each event (Austin 1999). Measured in logarithms of base 2, the unit of $H$ is bit. We will base our indices on what we will call the "reciprocal" of $H, 2^{\mathrm{H}}$, which can be thought of as the number of events occurring in equal proportion that would produce the same value of $H$. This reciprocal has the desirable property of recovering the original units (the number of events), however it is now a real number. In ecological literature, events usually involve species and cases involve individuals-or some measure of abundance; in our context, an "event" usually refers to a taxon and a "case", to the flow of biomass to or from a taxon. We calculated quantitative properties using the programming language Microsoft Visual $\mathrm{C}++$, checked the results in spreadsheets, and developed Microsoft Excel functions for most properties.

\section{Species properties}

Taxa can be classified as top, intermediate, and basal with quantitative information on trophic links in the same way as with qualitative data. We suggest a positional index $d$, which uses quantitative information about flux of biomass to assess to what degree a taxon $k$ is top, intermediate, or basal. For each taxon, one can measure the diversity of the biomass coming from its prey $\left(H_{\mathrm{N}}\right.$, the diversity of inflows) and of that going to its consumers $\left(H_{\mathrm{P}}\right.$, the diversity of outflows); for taxon $k$,

$$
\begin{aligned}
& H_{\mathrm{N}, k}=-\sum_{i=1}^{s} \frac{b_{i k}}{b_{\cdot k}} \log _{2} \frac{b_{i k}}{b_{\cdot k}} \\
& H_{\mathrm{P}, k}=-\sum_{j=1}^{s} \frac{b_{k j}}{b_{k} \cdot} \log _{2} \frac{b_{k j}}{b_{k} .}
\end{aligned}
$$

Column sum $b_{\cdot k}$ and row sum $b_{k}$. represent the total amount of biomass going to and emanating from taxon $k$, respectively. The reciprocals of $H_{\mathrm{N}, k}$ and $H_{\mathrm{P}, k}$ are

$$
\begin{aligned}
& n_{\mathrm{N}, k}= \begin{cases}2^{H_{\mathrm{N}, k}} & \\
0 & \text { if } b_{\cdot k}=0\end{cases} \\
& n_{\mathrm{P}, k}= \begin{cases}2^{H_{\mathrm{P}, k}} & \\
0 & \text { if } b_{k}=0 .\end{cases}
\end{aligned}
$$

These equivalent numbers of prey $\left(n_{\mathrm{N}}\right)$ and consumers $\left(n_{\mathrm{P}}\right)$ are used to compute a simple positional index $d_{k}^{\prime}$, which takes the disequitability in link magnitude of inflows to and outflows from taxon $k$ into account:

$$
d_{k}^{\prime}=\frac{n_{\mathrm{N}, k}}{n_{\mathrm{N}, k}+n_{\mathrm{P}, k}} .
$$

However, this index gives the same weight to both sets of prey and consumers, disregarding the magnitude of incoming $\left(b_{\cdot k}\right)$ and outgoing $\left(b_{k}\right.$ ) flows. To better capture the functional status of a taxon in a food web, the total amount of biomass coming from the prey and going to the consumers is taken into account. Consequently, the proposed index for taxon $k$ is

$$
d_{k}=\frac{b_{\cdot k} n_{\mathrm{N}, k}}{b_{\cdot k} n_{\mathrm{N}, k}+b_{k \cdot} n_{\mathrm{P}, k}} .
$$

Note that we use the prime for the unweighted version of indices (here $d_{k}^{\prime}$ ), which are those giving the same weight to each taxon irrespective of their inflows and outflows. With both indices, a taxon is top if $d_{k}=1$, basal if $d_{k}=0$, and intermediate otherwise. Computing the proportions of basal, intermediate, and top taxa is similar to finding the proportions of $d_{k}$ values equal to $0,>0$ and $<1$, and $=1$, respectively. However, we feel that a more sensible way of describing the positional status of the taxa in the food web would be to widen the interval of the top taxa from [1] to, e.g., [0.99,1], 
or more informatively to compute the frequency distribution of $d_{k}$ and $d_{k}^{\prime}$ values among all taxa.

The ratio of prey to consumers $(\mathrm{N}: \mathrm{P})$ can be expressed quantitatively $(\mathrm{q})$ by using the equivalent numbers of prey $\left(n_{\mathrm{N}}\right)$ and consumers $\left(n_{\mathrm{P}}\right)$. The total number of consumers is the count of nonzero $n_{\mathrm{N}}$. The diversities are computed over the $n_{\mathrm{N}}$ values, and their reciprocals correspond to the number of consumers that would populate the food web if each consumer had the same number of prey (strictly speaking, of prey equivalents $n_{\mathrm{N}}$ ). The same approach is applied to the $n_{\mathrm{P}}$ values to obtain the adjusted total number of prey. Taking the biomass of the fluxes into account requires a weighting of $n_{\mathrm{N}, k}$ by $b_{\cdot k}$, and of $n_{\mathrm{P}, k}$ by $b_{k}$. The two versions of the $\mathrm{N}: \mathrm{P}$ index are consequently expressed as follows

$$
\begin{aligned}
& \mathrm{N}: \mathrm{P}_{\mathrm{q}}^{\prime}=\frac{2^{\left[-\sum_{k=1}^{s}\left(\frac{n_{\mathrm{P}, k}}{\sum n_{\mathrm{P}, k}}\right) \times \log _{2}\left(\frac{n_{\mathrm{P}, k}}{\sum n_{\mathrm{P}, k}}\right)\right]}}{2^{\left[-\sum_{k=1}^{s}\left(\frac{n_{\mathrm{N}, k}}{\sum n_{\mathrm{N}, k}}\right) \times \log _{2}\left(\frac{n_{\mathrm{N}, k}}{\sum n_{\mathrm{N}, k}}\right)\right]}} \\
& \mathrm{N}: \mathrm{P}_{\mathrm{q}}=\frac{2^{\left[-\sum_{k=1}^{s}\left(\frac{b_{k} \cdot n_{\mathrm{P}, k}}{\sum b_{k} \cdot n_{\mathrm{P}, k}}\right) \times \log _{2}\left(\frac{b_{k \cdot} n_{\mathrm{P}, k}}{\sum b_{k \cdot} n_{\mathrm{P}, k}}\right)\right]}}{2^{\left[-\sum_{k=1}^{s}\left(\frac{b_{\cdot k} n_{\mathrm{N}, k}}{\sum b_{\cdot k} n_{\mathrm{N}, k}}\right) \times \log _{2}\left(\frac{b_{\cdot k} n_{\mathrm{N}, k}}{\sum b_{\cdot k} n_{\mathrm{N}, k}}\right)\right]}} .
\end{aligned}
$$

\section{Link properties}

First, one should note that the qualitative link density (LD) can be computed as the mean number of prey taxa over all species, or as the mean number of consumer taxa. We use both equivalences to devise an adequate way of expressing the link density in a quantitative manner, by computing the averages of the equivalent numbers of prey $n_{\mathrm{N}, k}$ and of consumers $n_{\mathrm{P}, k}$. The unweighted index $\mathrm{LD}_{\mathrm{q}}^{\prime}$, which gives the same weight to all taxa, is then:

$$
\begin{aligned}
\mathrm{LD}_{\mathrm{q}}^{\prime} & =\frac{1}{2}\left(\sum_{k=1}^{s} \frac{1}{s} n_{\mathrm{P}, k}+\sum_{k=1}^{s} \frac{1}{s} n_{\mathrm{N}, k}\right) \\
& =\frac{1}{2 \cdot s}\left(\sum_{k=1}^{s} n_{\mathrm{P}, k}+\sum_{k=1}^{s} n_{\mathrm{N}, k}\right) .
\end{aligned}
$$

The weighted index $\mathrm{LD}_{\mathrm{q}}$ requires a weighting of the species by their relative outflows and inflows:

$$
\mathrm{LD}_{\mathrm{q}}=\frac{1}{2}\left(\sum_{k=1}^{s} \frac{b_{k \cdot}}{b_{. .}} n_{\mathrm{P}, k}+\sum_{k=1}^{s} \frac{b_{\cdot k}}{b_{\cdot .}} n_{\mathrm{N}, k}\right) .
$$

Ulanowicz and Wolff (1991) have defined the effective connectance per node, $m$, which was proposed as a good candidate for a quantitative substitute of the link-density property (Bersier et al. 1999). The effective connectance per node is the reciprocal of the average diversity over inputs and outputs, which can be written as

$$
m=2^{\Phi / 2}
$$

with

$$
\Phi=\sum_{k=1}^{s} \frac{b_{k \cdot}}{b_{. .}} H_{\mathrm{P}, k}+\sum_{k=1}^{s} \frac{b_{\bullet k}}{b_{. \cdot}} H_{\mathrm{N}, k}
$$

The index $\Phi$ is the sum of the diversity of outflows weighted by the total outflows, and of the diversity of inflows weighted by the total inflows. $\Phi$ can be thought of as the average amount of choice in trophic pathways (Ulanowicz and Wolff 1991). We can expand the equation of $\mathrm{LD}_{\mathrm{q}}$ in the following form to see that the difference from $m$ resides solely in the weighting, which applies in our case not to the inflow and outflow diversities, but directly to the taxa's equivalent numbers of prey and consumers:

$$
\mathrm{LD}_{\mathrm{q}}=\frac{1}{2}\left(\sum_{k=1}^{s} \frac{b_{k \cdot}}{b_{. .}} 2^{H_{\mathrm{P}, k}}+\sum_{k=1}^{s} \frac{b_{\cdot k}}{b_{. .}} 2^{H_{\mathrm{N}, k}}\right) .
$$

Ulanowicz and Wolff (1991) also defined the topological connectance per node, $m^{*}$, which is the value that $m$ would take on if all nonzero $b_{i j}$ in the food web were assumed equal in magnitude; $m$ is always smaller or equal to $m^{*}$. As with the topological connectance per node, one can define the "topological" equivalents $\mathrm{LD}_{\mathrm{q}}^{*}$ and $\mathrm{LD}_{\mathrm{q}}^{\prime *}$ for which all links are given the same magnitude. We find that $\mathrm{LD}_{\mathrm{q}}^{\prime *}$ has the desirable feature of being equal to the qualitative link density LD. For a qualitative food web, directed connectance $(C)$ can be computed as $\mathrm{LD} / s$. With quantitative data, one can define connectance $C_{\mathrm{q}}$ in a similar way as $\mathrm{LD}_{\mathrm{q}} / s$, and $C_{\mathrm{q}}^{\prime}$ as $\mathrm{LD}_{\mathrm{q}}^{\prime} / s$.

Qualitative descriptors customarily used to categorize links are the proportions of links between top, intermediate, and basal taxa, namely \%T-I, \% T-B, \%II, and \%I-B. Above, we proposed two "positional" indices (Eqs. 9 and 10) to render the functional status of the taxa in a community, and suggested the possibility of more categories being defined from these indices than just basal, intermediate, and top. Whatever the convention adopted (more categories and/or categories defined with different limits), one can compute a quantitative equivalent of the proportions of links between two categories of taxa, A (the prey) and B (the consumers), in the following way:

$$
\% \mathrm{AB}_{\mathrm{q}}=\frac{\Phi_{\mathrm{AB}}}{\Phi}
$$

with

$$
\begin{aligned}
\Phi_{\mathrm{AB}}= & \sum_{k=1}^{\alpha} \frac{b_{i_{k}}}{b_{. .}}\left(-\sum_{n=1}^{\beta} \frac{b_{i_{k} j_{n}}}{b_{i_{k \cdot}}} \times \log _{2} \frac{b_{i_{k} j_{n}}}{b_{i_{k} \cdot}}\right) \\
& +\sum_{k=1}^{\beta} \frac{b_{\cdot j_{k}}}{b_{. \cdot}}\left(-\sum_{n=1}^{\alpha} \frac{b_{i_{n} j_{k}}}{b_{\bullet_{j_{k}}}} \times \log _{2} \frac{b_{i_{n} j_{k}}}{b_{\cdot_{j}}}\right) .
\end{aligned}
$$

$\Phi$, the overall average amount of choice in the trophic pathways, is measured as in Eq. $16 ; \Phi_{\mathrm{AB}}$ is similar to $\Phi$, but computed over the taxa belonging to categories $\mathrm{A}$ and $\mathrm{B}$ only (group $\mathrm{A}$ includes taxa $i_{1}, i_{2}, \ldots, i_{\alpha}$, and group B taxa $j_{1}, j_{2}, \ldots, j_{\beta}$ ), and with sums of rows 
$b_{i_{k}}$ and of columns $b_{\cdot_{j_{k}}}$ measured over all taxa. The value of $\% \mathrm{AB}_{\mathrm{q}}$ is the contribution of the links between taxa of groups A and B to $\Phi$; with the $s$ taxa of a food web distributed in two groups $\mathrm{A}$ and $\mathrm{B}$, we have $\Phi_{\mathrm{AB}}+\Phi_{\mathrm{BA}}$ $+\Phi_{\mathrm{AA}}+\Phi_{\mathrm{BB}}=\Phi$. An unweighted version of the proportion of links between $\mathrm{A}$ and $\mathrm{B}\left(\% \mathrm{AB}_{\mathrm{q}}^{\prime}\right)$ can be measured simply by replacing the weightings $b_{i_{k}} . / b_{\text {.., }}$ $b_{\bullet_{j}} / b_{. .}, b_{k \cdot} / b_{. .}$, and $b_{\bullet_{k}} / b_{. .}$in Eqs. 16 and 18 by $1 / s$.

\section{Chain properties}

The length $\left(\mathrm{cl}_{c}\right)$ of food chain $c$ can be adjusted for the importance of each trophic link comprised in the chain in the following way:

$$
\mathrm{cl}_{\mathrm{q}, c}^{\prime}=2^{\left[-\sum_{l=1}^{n_{l}}\left(\frac{b_{c, l}}{\sum b_{c, l}}\right) \times \log _{2}\left(\frac{b_{c, l}}{\sum b_{c, l}}\right)\right]},
$$

with $n_{1}$ the number of links forming chain $c$, and $b_{c, l}$ the biomass flow of the $l$ th link of chain $c$. The corrected length $\mathrm{cl}_{\mathrm{q}, c}^{\prime}$ reaches the original value $\mathrm{cl}_{c}$ only if all fluxes of the chain are equal in magnitude. Eq. 19 gives the same importance to each maximal food chain, disregarding the total amount of biomass flowing through it. We suggest the following approach to take the magnitude of flows of a chain into account for the computation of the weighted quantitative chain length $\left(\mathrm{cl}_{\mathrm{q}, c}\right)$; for each maximal food chain $c$, we compute the average biomass per effective link $\bar{b}_{c}=\Sigma b_{c, l} / \mathrm{cl}_{\mathrm{q}, c}^{\prime}$.

The weighted quantitative chain length $\mathrm{cl}_{\mathrm{q}, c}$ is then

$$
\mathrm{cl}_{\mathrm{q}, c}=\left(\frac{\bar{b}_{c} n_{\mathrm{mfc}}}{\sum \bar{b}_{c}}\right) \mathrm{cl}_{\mathrm{q}, c}^{\prime}
$$

with $n_{\mathrm{mfc}}=$ the number of maximal food chains. As a measure of the importance of a maximal food chain, we use the average biomass $\bar{b}_{c}$ per effective link, and not the total amount of flows $\Sigma b_{c, l}$, because long chains would tend to have large values simply as they are summed over more $b_{c, l}$ than short chains. The weighted and unweighted quantitative equivalents of the mean, median, standard deviation, and maximum of the lengths of food chains are computed for the $\mathrm{cl}_{\mathrm{q}, k}$ and $\mathrm{cl}_{\mathrm{q}, k}^{\prime}$ values, respectively.

The formulation of a quantitative counterpart for the number of food chains is problematic because there is no unequivocal way of defining the importance of a chain. One could measure the reciprocal of the Shannon index for the $\mathrm{cl}_{\mathrm{q}, k}^{\prime}$ or $\mathrm{cl}_{\mathrm{q}, k}$ values, which would equate to the equivalent number of maximal food chains assuming they all have a similar length; another possibility is to assign a "transfer efficiency" value $e_{c}$ to each maximal food chain, $e_{c}$ being measured as

$$
e_{c}=\prod_{l=1}^{n_{1}} b_{c, l} \text {. }
$$

The equivalent number of maximal food chains is then the reciprocal of the Shannon index measured over the $e_{c}$ values. Other definitions built in a similar way are conceivable, for example using $\Sigma b_{c, l}$. We see, however, no legitimacy for preferring one of these measures, and feel that the number of maximal food chains is in essence a qualitative property that has no adequate quantitative equivalent.

\section{Omnivory properties}

Among the many ways of defining an index of omnivory that considers the quantitative nature of trophic links, we chose first to define an individual index of omnivory $o_{k}$, applied to each taxon $k$, which is based on the distribution of trophic levels among the prey of $k$. The trophic level of taxon $k$ is $t_{k}$, and $n_{t}$ is the number of prey taxa located at trophic level $t$; the index reads as follows:

$$
o_{k}^{\prime}=-1+2^{\left[-\sum_{t=1}^{t_{k}}\left(\frac{n_{t}}{\sum n_{t}}\right) \times \log _{2}\left(\frac{n_{t}}{\sum n_{t}}\right)\right] .} \text {. }
$$

Note that $o_{k}^{\prime}$ does not take the importance of the trophic links into account, and can be applied to qualitative data. A weighted version $o_{k}$ can take on the following form:

$$
o_{k}=-1+2^{\left[-\sum_{t=1}^{t_{k}}\left(\frac{b_{t}}{\sum b_{t}}\right) \times \log _{2}\left(\frac{b_{t}}{\sum b_{t}}\right)\right]},
$$

with $b_{t}$ the total amount of biomass passing from trophic level $t$ to taxon $k$. This index equals 0 if taxon $k$ is basal or if it consumes prey belonging to the same trophic level; it equals 1 if taxon $k$ consumes prey located at two different levels in equal proportion, and has an upper bound of $t_{k}-1$. An adequate index of omnivory for the whole food web, $O_{\mathrm{q}}$, is the average of the $o_{k}$ values over all taxa; the unweighted version $O_{\mathrm{q}}^{\prime}$ is the average of all $o_{k}^{\prime}$ values. Note that, unlike Goldwasser and Roughgarden's (1993) index of omnivory, we do not build our indices on the variability of chain lengths. In such a case, a consumer taxon "inherits" the variability of its prey, which, for example, renders omnivorous a monophagous taxon eating an omnivore.

It may seem desirable to base the individual quantitative omnivory properties on a quantitative definition of trophic level. Possibilities may be found by weighting the links by their proportional biomass (Yodzis 1989), by computing the "trophic position" of the taxa (Levine 1980), or by the use of the $\mathrm{cl}_{\mathrm{q}}$ values defined above. However, these will yield non-integer trophic levels, which in turn will raise a problem with the summation in Eqs. 22 and 23. Since we see no satisfactory answer to that difficulty, we base our indices of omnivory on the adequate and customarily used qualitative definition (i.e., 1 plus the length of the longest chain from the focal taxon to a basal taxon).

\section{Consumer-prey asymmetries}

Generality $G$ is the average number of prey taxa per consumer. A quantitative unweighted version $G_{\mathrm{q}}^{\prime}$ can be defined simply as the average number of effective 
prey taxa, $n_{\mathrm{N}}$, over all consumer taxa, or for the weighted version $G_{\mathrm{q}}$ as a weighted average:

$$
\begin{aligned}
G_{q}^{\prime} & =\frac{1}{n_{\mathrm{T}}+n_{\mathrm{I}}} \sum_{k=1}^{s} n_{\mathrm{N}, k} \\
G_{q} & =\sum_{k=1}^{s} \frac{b_{\bullet}}{b_{.}} n_{\mathrm{N}, k}
\end{aligned}
$$

with $n_{\mathrm{T}}$ and $n_{\mathrm{I}}$ the number of top and intermediate taxa, respectively. Quantitative versions of the vulnerability $V$ follow accordingly as:

$$
\begin{aligned}
V_{q}^{\prime} & =\frac{1}{n_{\mathrm{I}}+n_{\mathrm{B}}} \sum_{k=1}^{s} n_{\mathrm{P}, k} \\
V_{q} & =\sum_{k=1}^{s} \frac{b_{k \cdot}}{b_{.} .} n_{\mathrm{P}, k}
\end{aligned}
$$

with $n_{\mathrm{B}}$ the number of basal taxa. Quantitative versions of the standard deviation of normalized generality, and of normalized vulnerability, require a standardization of the equivalent numbers of prey $n_{\mathrm{N}}$, and of consumers $n_{\mathrm{P}}$, respectively (Martinez and Williams 2000). This is achieved in the following way:

$$
\begin{aligned}
& g_{k}^{\prime}=\frac{s}{\sum_{k=1}^{s} n_{\mathrm{N}, k}} \times n_{\mathrm{N}, k} \\
& g_{k}=\frac{s}{\sum_{k=1}^{s} b_{\cdot k} n_{\mathrm{N}, k}} \times b_{\cdot k} n_{\mathrm{N}, k}
\end{aligned}
$$

with $g_{k}^{\prime}$ the unweighted, and $g_{k}$ the weighted standardized generality of taxon $k$. The standard deviations $\operatorname{sd}_{\mathrm{q}}^{\prime}$ and $\operatorname{sd} G_{\mathrm{q}}$ are then computed with these standardized values. The unweighted and weighted standardized vulnerability for taxon $k$ ( $v_{k}^{\prime}$ and $v_{k}$ ) follow accordingly. Note that Levine (1980) has defined indices of trophic specialization, computed for each taxon, which are especially interesting if loops are present. They are based on the variance of weighted path lengths for a taxon, or on the variance of the trophic positions of the prey of a taxon.

\section{An Example Application}

The results of applying the various food-web properties defined above to the Chesapeake Bay ecosystem (Appendix) are given in Table 1 for the taxon-specific indices, and in Table 2 for the food-web descriptors. The positional indices $d_{k}^{\prime}$ and $d_{k}$ (Table 1) indicate to what extent a taxon $(k)$ is basal (value of 0 ) or top (value of 1). We observe an important difference between the unweighted $\left(d_{k}^{\prime}\right.$, where the same weight is given to each taxon) and the weighted indices ( $d_{k}$, where the total amount of flows to and from a taxon are taken into account). This reflects the fact that inflows to a taxon are generally much larger than the outflows, which is consistent with trophodynamics (Lindeman 1942, Odum 1971). However, that is not a mandatory feature, especially in small and open sys- tems where allochthonous inputs are important (Polis and Hurd 1996). These differences in the distribution of $d$ values is reflected in the unequal percentages of top $(\% T)$ and intermediate $(\% I)$ taxa when computed using the $d_{k}$ or $d_{k}^{\prime}$ values, i.e., $\% T_{\mathrm{q}}^{\prime} \neq \% T_{\mathrm{q}}$, and $\% I_{\mathrm{q}}^{\prime} \neq$ $\% I_{\mathrm{q}}$ (Table 2). Using 0.99 as the lower limit for top taxa, we find that three taxa (taxon 19, blue crab; taxon 21 , alewife and blue herring; taxon 27 , spot) increase the proportion of top taxa from 0.36 for the unweighted to 0.45 for the weighted version. Taxon 14 (other polychaetes) witnesses the highest jump between the unweighted and the weighted index. Polychaetes prey on a single taxon (taxon 3, sediment bacteria) and are consumed by five fish species; however, the inflows outweigh the outflows to such an extent that $d$ rises close to the 0.99 limit.

The qualitative ratio of prey to consumers (N:P) equals 0.75 , meaning that there is an average of four consumer taxa for three prey taxa. This may be an intuitively strange result; however it can be explained for the present food web by the difference in taxonomic resolution between invertebrates on the one hand, and fishes on the other. While the former, which make up the bulk of the prey taxa, are often taxonomically highly aggregate, the latter dominate in their role as consumers and are often resolved at the species level. Due to a slightly stronger disequitability in the distribution of the equivalent number of consumers, the unweighted quantitative index $\mathrm{N}: \mathrm{P}_{\mathrm{q}}^{\prime}$ yields 0.71 . The weighted version $\mathrm{N}: \mathrm{P}_{\mathrm{q}}$ is 0.32 , which reveals a much higher disequitability in the distribution of the equivalent numbers of consumers once multiplied by the outflows, i.e., the distribution of $b_{\cdot k} n_{\mathrm{N}, k}$ is much more even than that of $b_{k}, n_{\mathrm{P}, k}$, which increases the numerator of Eq. 12. A closer inspection of the original data set (Appendix) shows that this is mainly due to the sediment bacteria, which are, by their huge biomass and large number of consumers, the dominant prey taxon in the system.

The qualitative link density LD for the Chesapeake Bay ecosystem is 2.21 , meaning that each taxon has on average 2.21 prey and 2.21 consumers. This value falls to 1.37 when computed as the average of the effective numbers of prey and consumers, $\mathrm{LD}_{\mathrm{q}}^{\prime}$. It can be shown that $\mathrm{LD}_{\mathrm{q}}^{\prime}$ is always smaller than or equal to $\mathrm{LD}$, and this decrease reflects the degree of departure from a uniform distribution in flows from prey and to consumers. The weighted quantitative link density $\mathrm{LD}_{\mathrm{q}}$ reaches a value of 2.1 , which indicates that taxa having high total inflows $b_{\bullet k}$ and/or outflows $b_{k}$. also have high $n_{\mathrm{N}, k}$ and/or $n_{\mathrm{P}, k}$ values. Accordingly, the weighted and unweighted quantitative connectance follow a similar trend.

As for the proportion of top, intermediate, and basal taxa, the unweighted quantitative proportions of links $\% \mathrm{~T}-\mathrm{I}_{\mathrm{q}}^{\prime}, \% \mathrm{~T}-\mathrm{B}_{\mathrm{q}}^{\prime}, \% \mathrm{I}-\mathrm{I}_{\mathrm{q}}^{\prime}$, and \%I-B $\mathrm{B}_{\mathrm{q}}^{\prime}$ are not markedly different from their qualitative counterparts (Table 2). This reflects similar distributions of link magnitude in the four groups, so that their contributions to $\Phi$, the overall average amount of choice in the trophic path- 
TABle 1. Taxon-specific qualitative and quantitative descriptors for the Chesapeake Bay mesohaline ecosystem (see the Appendix).

\begin{tabular}{|c|c|c|c|c|c|c|c|c|c|c|c|c|c|c|}
\hline \multirow{4}{*}{$\begin{array}{c}\text { Taxon, } \\
k\end{array}$} & \multirow{2}{*}{\multicolumn{2}{|c|}{$\begin{array}{l}\text { Qualita- } \\
\text { tive } \\
\end{array}$}} & \multicolumn{2}{|c|}{ Marginal sums } & \multicolumn{10}{|c|}{ Quantitative indices } \\
\hline & & & \multirow{3}{*}{$\begin{array}{c}\text { Sum of } \\
\text { inflows, } \\
b_{\cdot k}\end{array}$} & \multirow{3}{*}{$\begin{array}{c}\text { Sum of } \\
\text { outflows, } \\
b_{k} .\end{array}$} & \multicolumn{2}{|c|}{ Effective no. } & \multirow{2}{*}{\multicolumn{2}{|c|}{$\begin{array}{l}\text { Positional } \\
\text { index }\end{array}$}} & \multirow{2}{*}{\multicolumn{2}{|c|}{$\begin{array}{l}\text { Index of } \\
\text { omnivory }\end{array}$}} & \multirow{2}{*}{\multicolumn{2}{|c|}{$\begin{array}{l}\text { Standardized } \\
\text { generality }\end{array}$}} & \multirow{2}{*}{\multicolumn{2}{|c|}{$\begin{array}{l}\text { Standardized } \\
\text { vulnerability }\end{array}$}} \\
\hline & No. & No. & & & Prey, & Pred., & & & & & & & & \\
\hline & & & & & $n_{\mathrm{N}, k}$ & $n_{\mathrm{P}, k}$ & $d_{k}^{\prime}$ & $d_{k}$ & $o_{k}^{\prime}$ & $o_{k}$ & $g_{k}^{\prime}$ & $g_{k}$ & $v_{k}^{\prime}$ & $v_{k}$ \\
\hline 1 & 0 & 7 & 0 & 80051 & 0 & 3.19 & 0 & 0 & 0 & 0 & 0 & 0 & 2.62 & 5.47 \\
\hline 2 & 0 & 8 & 0 & 2977 & 0 & 3.21 & 0 & 0 & 0 & 0 & 0 & 0 & 2.64 & 0.21 \\
\hline 3 & 0 & 6 & 0 & 294955 & 0 & 3.63 & 0 & 0 & 0 & 0 & 0 & 0 & 2.99 & 22.96 \\
\hline 4 & 0 & 1 & 0 & 18086 & 0 & 1 & 0 & 0 & 0 & 0 & 0 & 0 & 0.82 & 0.39 \\
\hline 5 & 0 & 1 & 0 & 88721 & 0 & 1 & 0 & 0 & 0 & 0 & 0 & 0 & 0.82 & 1.90 \\
\hline 6 & 1 & 1 & 88721 & 31638 & 1 & 1 & 0.5 & 0.74 & 0 & 0 & 0.65 & 3.90 & 0.82 & 0.68 \\
\hline 7 & 3 & 5 & 64224 & 11742 & 2.13 & 2.42 & 0.47 & 0.83 & 0.89 & 1.00 & 1.39 & 6.00 & 2.00 & 0.61 \\
\hline 8 & 3 & 7 & 46389 & 9855 & 1.81 & 2.48 & 0.42 & 0.77 & 0.89 & 0.56 & 1.19 & 3.69 & 2.04 & 0.52 \\
\hline 9 & 3 & 1 & 10447 & 552 & 2.01 & 1 & 0.67 & 0.97 & 2 & 1.01 & 1.31 & 0.92 & 0.82 & 0.01 \\
\hline 10 & 2 & 0 & 1711 & 0 & 1.88 & 0 & 1 & 1 & 1 & 0.88 & 1.23 & 0.14 & 0 & 0 \\
\hline 11 & 3 & 1 & 4594 & 538 & 1.41 & 1 & 0.58 & 0.92 & 0.89 & 0.27 & 0.92 & 0.28 & 0.82 & 0.01 \\
\hline 12 & 3 & 2 & 2488 & 224 & 1.41 & 1.18 & 0.54 & 0.93 & 0.89 & 0.26 & 0.92 & 0.15 & 0.97 & 0.01 \\
\hline 13 & 3 & 0 & 4830 & 0 & 1.41 & 0 & 1 & 1 & 0.89 & 0.26 & 0.92 & 0.30 & 0 & 0 \\
\hline 14 & 1 & 5 & 160831 & 609 & 1 & 3.41 & 0.23 & 0.99 & 0 & 0 & 0.65 & 7.06 & 2.80 & 0.04 \\
\hline 15 & 1 & 6 & 25062 & 547 & 1 & 2.94 & 0.25 & 0.94 & 0 & 0 & 0.65 & 1.10 & 2.42 & 0.03 \\
\hline 16 & 1 & 2 & 57335 & 4593 & 1 & 1.07 & 0.48 & 0.92 & 0 & 0 & 0.65 & 2.52 & 0.88 & 0.11 \\
\hline 17 & 2 & 0 & 54048 & 0 & 1.89 & 0 & 1 & 1 & 0 & 0 & 1.24 & 4.49 & 0 & 0 \\
\hline 18 & 1 & 6 & 14075 & 1027 & 1 & 1.31 & 0.43 & 0.91 & 0 & 0 & 0.65 & 0.62 & 1.08 & 0.03 \\
\hline 19 & 7 & 2 & 8593 & 295 & 4.12 & 1.05 & 0.80 & 0.99 & 2.59 & 1.48 & 2.69 & 1.55 & 0.86 & 0.01 \\
\hline 20 & 1 & 0 & 4.9 & 0 & 1 & 0 & 1 & 1 & 0 & 0 & 0.65 & $<0.01$ & 0 & 0 \\
\hline 21 & 1 & 1 & 26 & 0.2 & 1 & 1 & 0.5 & 0.99 & 0 & 0 & 0.65 & $<0.01$ & 0.82 & $<0.01$ \\
\hline 22 & 3 & 6 & 1823 & 153 & 1.59 & 3.58 & 0.31 & 0.84 & 0.89 & 0.55 & 1.04 & 0.13 & 2.94 & 0.01 \\
\hline 23 & 3 & 3 & 273 & 21 & 1.42 & 2.64 & 0.35 & 0.87 & 0.89 & 0.36 & 0.93 & 0.02 & 2.17 & $<0.01$ \\
\hline 24 & 1 & 0 & 5 & 0 & 1 & 0 & 1 & 1 & 0 & 0 & 0.65 & $<0.01$ & 0 & 0 \\
\hline 25 & 3 & 0 & 10 & 0 & 1.93 & 0 & 1 & 1 & 0 & 0 & 1.26 & $<0.01$ & 0 & 0 \\
\hline 26 & 4 & 0 & 96 & 0 & 2.95 & 0 & 1 & 1 & 0.75 & 0.36 & 1.93 & 0.01 & 0 & 0 \\
\hline 27 & 5 & 1 & 483 & 10 & 2.66 & 1 & 0.72 & 0.99 & 0.65 & 0.14 & 1.74 & 0.06 & 0.82 & $<0.01$ \\
\hline 28 & 3 & 0 & 150 & 0 & 2.56 & 0 & 1 & 1 & 0.89 & 0.38 & 1.67 & 0.02 & 0 & 0 \\
\hline 29 & 3 & 0 & 219 & 0 & 2.24 & 0 & 1 & 1 & 0 & 0 & 1.46 & 0.02 & 0 & 0 \\
\hline 30 & 3 & 0 & 16 & 0 & 2.41 & 0 & 1 & 1 & 0.89 & 0.9 & 1.58 & $<0.01$ & 0 & 0 \\
\hline 31 & 1 & 1 & 91 & 4 & 1 & 1 & 0.5 & 0.96 & 0 & 0 & 0.65 & $<0.01$ & 0.82 & $<0.01$ \\
\hline 32 & 4 & 0 & 26 & 0 & 3.11 & 0 & 1 & 1 & 1.83 & 0.82 & 2.03 & $<0.01$ & 0 & 0 \\
\hline 33 & 4 & 0 & 30 & 0 & 2.52 & 0 & 1 & 1 & 0 & 0 & 1.65 & $<0.01$ & 0 & 0 \\
\hline
\end{tabular}

Notes: Taxon numbers refer to those of the food-web matrix in the Appendix. (In general, high numbers are near the top of the food web.) Quantitative indices come in two versions: unweighted (denoted by the "prime"), where the same weight is given to each taxon; and weighted, where the total amount of flows from and to the taxon are taken into account.

ways, remains essentially unaffected. Deviations from the qualitative proportions are particularly manifest in a higher value for $\% \mathrm{~T}-\mathrm{I}_{\mathrm{q}}^{\prime}$ and a lower value for $\% \mathrm{I}-\mathrm{B}_{\mathrm{q}}^{\prime}$, which highlights a more equitable distribution of link magnitudes between top and intermediate taxa than between intermediate and basal taxa. The results change markedly with the weighted proportions $\% \mathrm{~T}-\mathrm{I}_{\mathrm{q}}, \% \mathrm{~T}-\mathrm{B}_{\mathrm{q}}$ $\% \mathrm{I}-\mathrm{I}_{\mathrm{q}}$, and $\% \mathrm{I}-\mathrm{B}_{\mathrm{q}}$, which again reflect trophodynamics.

There is a total of 217 food chains in the Chesapeake Bay ecosystem, of which 134 are maximal ones. Note that there is no loop in the food-web graph, except for a cannibalistic one (taxon 19, Fig. 1). The unweighted quantitative properties describing the maximal food chains always produce lower values than their qualitative counterparts (only maximal food chains [those that link a top and a basal taxon] are treated in Table $2)$. This is an expected feature since the "effective" chain length attains the qualitative one only if the flows contained in the chain are equal in magnitude. The weighted quantitative average chain length $M_{\mathrm{cl}, \mathrm{q}}$ is smaller than the unweighted $M_{\mathrm{cl}, \mathrm{q}}^{\prime}$, indicating that, on average, long chains are less important in term of average biomass than short chains.

In the Chesapeake Bay ecosystem, $45 \%$ of the taxa are omnivorous. Our unweighted index of omnivory $O_{\mathrm{q}}^{\prime}$ comes close to this figure, with a value of 0.51 . Inspection of the taxon-specific indices of omnivory $o_{k}^{\prime}$ (Table 1) shows that most omnivorous taxa consume prey in unequal proportions from two trophic levels, in which case $o_{k}^{\prime}<1$. Three taxa consume on more than two trophic levels, of which the blue crabs (taxon 19) has the widest regime. The value of most taxon-specific indices $o_{k}$ is lower than their unweighted counterpart: the disequitability is higher once the biomass coming from the various trophic levels is taken into account, since omnivorous taxa eat preferentially from one trophic level, and consequently the value of the weighted quantitative omnivory index $O_{\mathrm{q}}$ falls to 0.28 . Microzooplankton (taxon 7) forms an exception, consuming prey in very equal proportions from two trophic levels.

The qualitative generality $G$ for the Chesapeake data set shows that each consumer taxon feeds on average 
TABLE 2. Values of the qualitative and quantitative descriptors for the Chesapeake Bay mesohaline ecosystem (see Appendix).

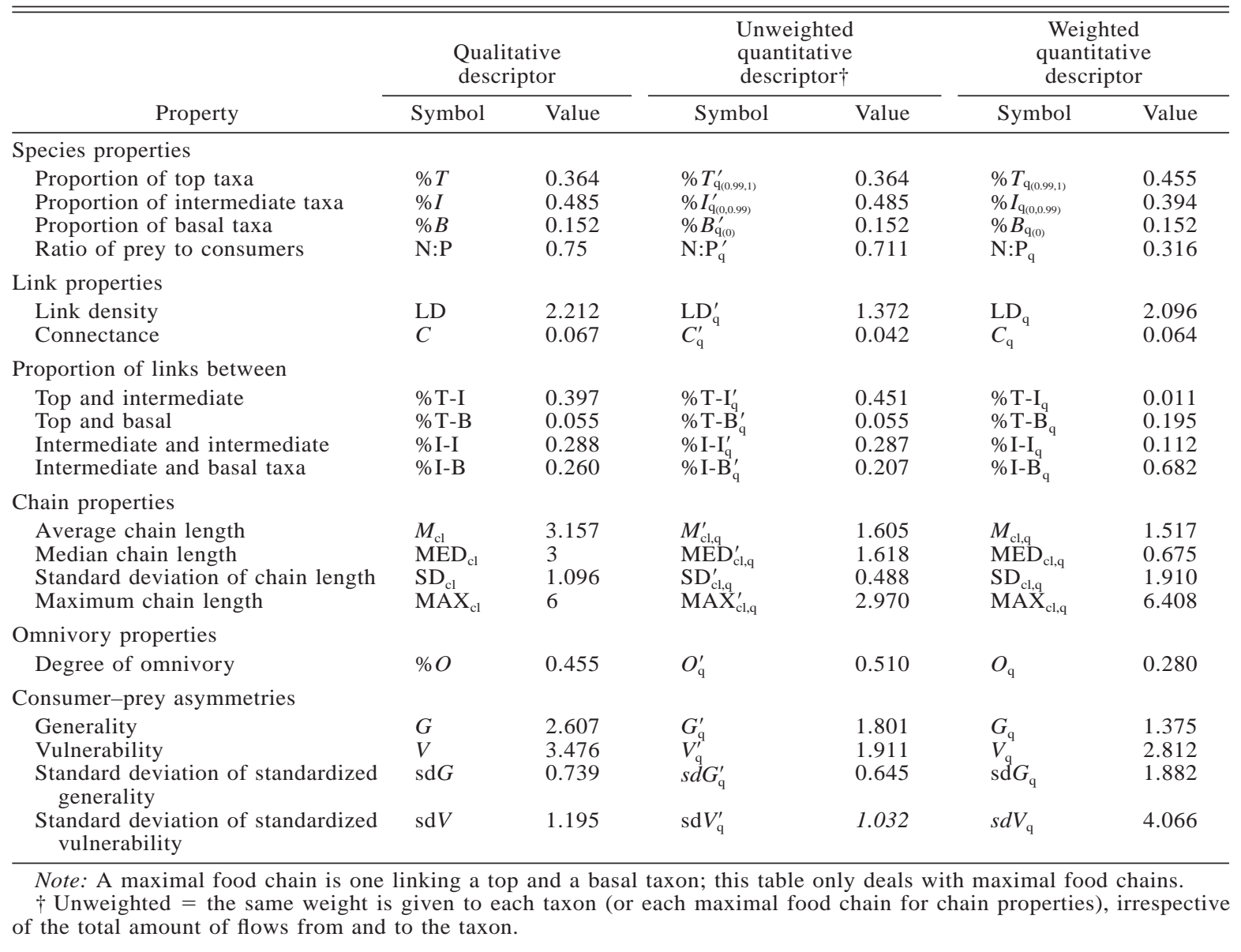

on 2.61 prey taxa. Once computed over the effective number of prey, this index decreases to 1.8 , and further drops down to 1.38 when weighted by the outflows. When considering the vulnerability $V$, we find that each prey taxon has on average 3.48 consumers, and that this value decreases to 1.91 with the unweighted quantitative index $V_{\mathrm{q}}^{\prime}$. It is noteworthy that prey taxa have more consumers than consumer taxa have prey, but that this inequality is reversed once one considers the effective numbers of consumers and prey. It is further interesting to note that, in contrast to a comparison of the respective quantitative measures of generality $\left(G_{\mathrm{q}}^{\prime}\right.$ and $G_{\mathrm{q}}$ ) the weighted vulnerability $\left(V_{\mathrm{q}}\right)$ is much larger than the unweighted one $\left(V_{\mathrm{q}}^{\prime}\right)$. This indicates that important prey taxa in term of outflows are also those with the highest number of consumer taxa, and that the contrary is true for consumer taxa. The variability in number of prey and of consumers is measured by the standard deviation of generality $(\operatorname{sd} G)$ and of vulnerability $(\operatorname{sd} V)$, respectively. Note also that the weighted quantitative $\operatorname{sd} G_{\mathrm{q}}$ and $\mathrm{sd} V_{\mathrm{q}}$, are much higher than their respective qualitative and unweighted quantitative versions. This indicates a strong variability once total in- flows and outflows are taken into account. The standardized generality and vulnerability (Table 1) show which taxa are responsible for this increase in inequitability - the polychaetes (taxon 14), the microzooplankton (taxon 7), and the meiofauna (taxon 17) on the side of the consumers; the sediment bacteria (taxon 3 ), and to a lesser extent the phytoplankton (taxon 1) on the side of the prey. Again, the higher $\operatorname{sd} V_{\mathrm{q}} \operatorname{com}$ pared to $\mathrm{sd} G_{\mathrm{q}}$ is consistent with the difference between quantitative vulnerability and generality.

\section{DISCUSSION}

The purpose of formulating quantitative properties is to gain ecological information from a weighted food web. The qualitative approach collapses the quantitative data of the Chesapeake Bay ecosystem (see the Appendix) into its bare connective structure, which is pictured in Fig. 1. From such a graph, one would for example assume that taxon 21 (alewife and herring) is a typical "intermediate" taxon, with one prey and one consumer; our positional index $d$ (Table 1) indicates, however, that it is functionally very close to a top taxon, being preyed upon only marginally by taxon 33 (striped 
bass). Taxon 9 (ctenophores) is seen as an important omnivorous species, eating prey at three different trophic levels; this is indeed captured by our unweighted omnivory index $\left(o^{\prime}\right)$-but once the magnitude of inflows are taken into account, the value of the index $(o)$ drops, reflecting the fact that only $1 \%$ of the inflows to ctenophores come from trophic level 1 . The weighted indices of standardized generality $(g)$ and vulnerability (v) may seem to yield puzzling results. For example, taxon 14 (other polychaetes) has a $g$ value of 7.06 and preys upon a single taxon, while taxon 32 (summer flounder) has four prey and a $g$ value close to 0 . This apparent inconsistency is explained by the huge differences in magnitude of inflows between low- and high-trophic-level taxa, which is intentionally taken into account by the weighted index. The usual concepts of generality and vulnerability are suitably expressed by the unweighted indices $g^{\prime}$ and $v^{\prime}$, respectively. Overall, our taxon-specific indices appear to correctly capture the quantitative information of trophic interactions. Synthetic properties of food webs shed light on more-hidden characteristics of food webs. Those built up from the individual descriptors (species and omnivory properties, predator-prey asymmetries) are expected to carry with them the biological realism seen in the taxon-specific indices. We believe that the other global descriptors (link and chain properties) suitably incorporate the quantitative information. For example, the fact that the unweighted link density $\left(\mathrm{LD}_{\mathrm{q}}^{\prime}\right)$ yields the same result as its qualitative counterpart (LD) when applied to qualitative food webs supports this view.

For each qualitative measure, we propose both a socalled unweighted version, where each taxon (or food chain) is given the same value, and a weighted version, where total inflows and outflows are taken into account. We believe that there is no primacy of using weighted quantitative, unweighted quantitative, or qualitative properties, but that it is their combined use that provides the best insight into the studied system, as demonstrated in the Results section, above. However, these three categories of descriptors do not behave alike with regard to varying levels of sampling effort. Our preliminary results of sensitivity tests show that a stronger sampling effort is required for most qualitative properties to attain their original values than for the unweighted quantitative versions, and that weighted quantitative measures are those reaching an asymptote the fastest (C. Banašek-Richter, M.-F. Cattin, and L.-F. Bersier, unpublished manuscript). Note that it has already been shown how qualitative properties vary with sampling effort (Goldwasser and Roughgarden 1997, Bersier et al. 1999, Martinez et al. 1999). In sum, quantitative properties are not only useful as bearers of ecological information, they also represent a much more robust description of weighted matrices. Such quantitative data have been advocated by many (e.g., May 1983, Kenny and Loehle 1991, Cohen et al. 1993), and are now becoming available (e.g., Winemiller 1990, Ulanowicz and Wolff 1991, Goldwasser and Roughgarden
1993, Memmot et al. 1994, de Ruiter 1995, Tavares-Cromar and Williams 1996, Omacini et al. 2001).

We devised the computation of our descriptors on information-based indices. There are probably infinite possibilities for using quantitative information to compute food-web properties. A simple and natural approach is to calculate the qualitative properties at various levels of link importance (Winemiller 1990). We chose information-theoretical indices because they allow finding the number of elements that would give a similar value of the same index given that these elements occur in equal proportion (what we call the "reciprocal" of $H$; see Quantitative descriptors, above). Hence the original units (e.g., number of flows) are recovered, and it is then easier to construct a property that conforms with its original qualitative essence. However, while some conversions follow naturally (e.g., the quantitative link density, Eqs. 15 and 16), we found some properties to be more resistant to any evident formulation in quantitative terms - the epitome being the weighted quantitative number of maximal food chains, for which we found no satisfactory counterpart.

Instead of using Shannon's formula, other diversity measures, such as the Simpson index (Simpson 1949), or indices based on Rényi's generalized entropy formula (Hill 1973, Legendre and Legendre 1998), are conceivable. For each of these, it is possible to compute their corresponding "diversity number" (our reciprocal of $H$ ). They may be even less sensitive to varying levels of sampling effort as they give less weight to small proportions. Note however that Brillouin's formula (Pielou 1966a) is not applicable because of its inconsistency in respect to the units of $b_{i j}$ (the biomass passing from taxon $i$ to taxon $j$ per unit surface area and time). We tested the Simpson index in lieu of the Shannon index for the quantitative link-density property (Eqs. 15 and 16). Indeed, we found that it performed slightly better than the Shannon index, the original value being reached slightly earlier. However, a disadvantage is a higher variance of the estimate, with a CV about 10\% larger than with Shannon's index. Thus, the use of the Shannon index appears to be a good compromise between accuracy and precision.

The use (and misuse) of diversity indices and especially of the Shannon index has raised much discussion in ecological literature (e.g., Pielou 1966b, Hurlbert 1971, Margalef 1995, Gotelli and Graves 1996). Nowadays, the use of the Shannon index as a measure of diversity per se is less popular among ecologists, and evenness measures are often preferred (e.g., Smith and Wilson 1996, Hofer and Bersier 2001). Indices based on information theory are, however, pertinent as phenomenological measures of growth and development for large systems (e.g., Ulanowicz 1986, Hirata 1995). The rationale for their suitability can be summarized as follows. As developing systems are changing, the "probabilities of microscopic events internal to maturing systems change accordingly. The study of what gives rise to a change in probability 
assignment defines the realm of information theory", (Ulanowicz 1986:81). The indices developed by these authors are derived in mathematically strict form from information theory; the overall average amount of choice in the trophic pathways $\Phi$ and the effective connectance $m$ (Ulanowicz and Wolff 1991) are excellent examples (Eqs. 15 and 16). In our case, the utilization of the Shannon index follows from more simple grounds, and is justified operationally as an adequate method for taking the quantitative nature of trophic links into account.

Qualitative properties have been analyzed as dependent variables against a measure of food web sizethe number of taxa, $s$-using various analytical techniques (e.g., Briand and Cohen 1984, Sugihara et al. 1989, Bengtsson 1994, Bersier and Sugihara 1997). There is much contention about the regularities found from such studies (e.g., Havens 1993, Martinez 1993 ), and we think that much of the disagreement has its roots in the sensitivity of qualitative properties to sampling effort (Bersier et al. 1999). We hope that the descriptors we propose will help settle these controversies. However, their interpretation is more difficult, since their value is a function of two components: the number of flows, and the shape of the relative frequency distribution of the magnitude of these flows. Thus, the same value of an index can be obtained from many flows if few flows dominate the frequency distribution, or from few flows with a more even distribution. Such indeterminacy can be resolved by comparing the values obtained with the corresponding qualitative property, or simply by examination of the distributions of link importance for each taxon.

A difficulty with the use of quantitative properties lies in the choice of a currency for the trophic interactions. Different approaches have been used: measures based on the quantity of biomass transferred (e.g., flow of carbon, Baird and Ulanowicz 1989; feeding rate, de Ruiter et al. 1995; volume, Winemiller 1990; index of relative importance, Tavares-Cromar and Williams 1996) are adequate for a "static" representation of web structure, while measures based on interaction strength (e.g., Paine 1992, Wootton 1994, Raffaelli and Hall 1996, Laska and Wootton 1998) and on the frequency of trophic interactions (Goldwasser and Roughgarden 1993) would better describe the "dynamical" aspect of web structure. As mentioned above, the units must be comparable row- and columnwise. This criterion is not fulfilled by the index of relative importance (Tavares-Cromar and Williams 1996), which gives the same weight to all consumers. It is also important to be aware that measures of interaction strength and of flow of biomass may yield quite different values for the same trophic interaction (Polis 1994). Thus, when comparative studies are undertaken, it is essential to respect the consistency in the units quantifying trophic interactions. With these constraints kept in mind, we believe that the tools proposed here will be useful to extract meaningful information from objects as complex as weighted food webs, and will help develop a deeper insight into the structure of quantitative food webs.

\section{ACKNOWLEDGMENTS}

We are grateful to R. Baltensperger, J. R. Bence, J. Chattopadhyay, D. Deb, J. P. Gabriel, C. Mermod, R. S. Sarkar, R. Ulanowicz, and an anonymous reviewer for their help and comments on the manuscript. This work was supported by Swiss NSF grant 31-52566.97, the Novartis Foundation, and partly the NCCR Plant Survival.

\section{Literature Cited}

Austin, M. P. 1999. A silent clash of paradigms: some inconsistencies in community ecology. Oikos 86:170-178.

Baird, D., and R. E. Ulanowicz. 1989. The seasonal dynamics of the Chesapeake Bay ecosystem. Ecological Monographs 59:329-364.

Bengtsson, J. 1994. Confounding variables and independent observations in comparative analyses of food webs. Ecology 75:1282-1288.

Bersier, L.-F., P. Dixon, and G. Sugihara. 1999. Scale-invariant or scale-dependent behavior of the link density property in food webs: A matter of sampling effort? American Naturalist 153:676-682.

Bersier, L.-F., and G. Sugihara. 1997. Scaling regions for food web properties. Proceedings of the National Academy of Sciences (USA) 94:1247-1251.

Briand, F., and J. E. Cohen. 1984. Community food webs have scale-invariant structure. Nature (London) 307:264267.

Carney, H. J., K. E. Havens, and L.-F. Bersier. 1997. Nonlinear scale dependence and spatiotemporal variability in planktonic food webs. Oikos 79:230-240.

Closs, G., G. A. Watterson, and P. J. Donnelly. 1995. Constant predator-prey ratios: An arithmetical artifact? Ecology 74: 238-243.

Cohen, J. E. 1977. Ratio of prey to predators in community food webs. Nature (London) 270:165-167.

Cohen, J. E. 1978. Food webs and niche space. Princeton University Press, Princeton, New Jersey, USA.

Cohen, J. E. 1989. Food webs and community structure. Pages 181-202 in J. Roughgarden, R. M. May, and S. A. Levin, editors. Perspectives in ecological theory. Princeton University Press, Princeton, New Jersey, USA.

Cohen, J. E., et al. 1993. Improving food webs. Ecology 74: 252-258

Cohen, J. E., and C. M. Newman. 1985. A stochastic theory of community food webs. I. Models and aggregated data. Proceedings of the Royal Society of London B 224:449-461.

de Ruiter, P. C., A. M. Neutel, and J. C. Moore. 1995. Energetics, patterns of interaction strengths, and stability in real ecosystems. Science 269:1257-1260.

Deb, D. 1995. Scale-dependence of food web structures: tropical ponds a paradigm. Oikos 72:245-262.

Goldwasser, L., and J. Roughgarden. 1993. Construction and analysis of a large Caribbean food web. Ecology 74:12161233.

Goldwasser, L., and J. Roughgarden. 1997. Sampling effects and the estimation of food-web properties. Ecology 78:4154.

Gotelli, N. J., and G. R. Graves. 1996. Null models in ecology. Smithsonian Institution Press, Washington, D.C., USA.

Hall, S. J., and D. Raffaelli. 1991. Food-web patterns: lessons from a species-rich web. Journal of Animal Ecology 60: $823-842$.

Havens, K. E. 1992. Scale and structure in natural food webs. Science 257:1107-1109.

Havens, K. E. 1993. Effect of scale on food web structureresponse. Science 260:243.

Hill, M. O. 1973. Diversity and evenness: a unifying notation and its consequences. Ecology 54:427-432. 
Hirata, H. 1995. Information theory and ecological networks. Pages 623-642 in B. C. Patten, S. E. Jorgensen, and S. I. Auerbach, editors. Complex ecology. The part-whole relation in ecosystems. Prentice Hall PTR, Englewood Cliffs, New Jersey, USA.

Hofer, U., and L.-F. Bersier. 2001. Herpetofaunal diversity and abundance in tropical upland forests of Cameroon and Panama. Biotropica 33:142-152.

Hurlbert, S. H. 1971. The nonconcept of species diversity: a critique and alternative parameters. Ecology 52:577-586.

Kenny, D., and C. Loehle. 1991. Are food webs randomly connected? Ecology 72:1794-1799.

Laska, M. S., and J. T. Wootton. 1998. Theoretical concepts and empirical approaches to measuring interaction strength Ecology 79:461-476.

Lawton, J. H. 1989. Food webs. Pages 43-78 in J. M. Cherrett, editor. Ecological concepts. Blackwell Scientific, Oxford, UK.

Legendre, P., and L. Legendre. 1998. Numerical ecology. Elsevier, Amsterdam, The Netherlands.

Levine, S. 1980. Several measures of trophic structure applicable to complex food webs. Journal of Theoretical Biology 83:195-207.

Lindeman, R. L. 1942. The trophic-dynamic aspect of ecology. Ecology 23:399-418.

Margalef, R. 1995. Information theory and complex ecology. Pages 40-50 in B. C. Patten, S. E. Jorgensen, and S. I. Auerbach, editors. Complex ecology. The part-whole relation in ecosystems. Prentice Hall PTR, Englewood Cliffs, New Jersey, USA.

Martinez, N. D. 1991. Artifacts or attributes? Effects of resolution on the Little Rock Lake food web. Ecological Monographs 61:367-392.

Martinez, N. D. 1992. Constant connectance in community food webs. American Naturalist 139:1208-1218.

Martinez, N. D. 1993. Effects of scale on food web structure. Science 260:242-243.

Martinez, N. D., B. A. Hawkins, H. A. Dawah, and B. P. Feifarek. 1999. Effects of sampling effort on characterization of food-web structure. Ecology 80:1044-1055.

Martinez, N. D., and J. H. Lawton. 1995. Scale and foodweb structure: from local to global. Oikos 73:148-154.

May, R. M. 1974. Stability and complexity of model ecosystems. Princeton University Press, Princeton, New Jersey, USA.

May, R. M. 1983. The structure of food webs. Nature 301: $566-568$.

Memmott, J., H. C. J. Godfray, and I. D. Gauld. 1994. The structure of a tropical host-parasitoid community. Journal of Animal Ecology 63:521-540.

Odum, E. 1971. Fundamentals of ecology. Saunders, Philadelphia, Pennsylvania, USA.

Omacini, M., E. J. Chaneton, C. M. Ghersa, and C. B. Müller 2001. Symbiotic fungal endophytes control insect hostparasite interaction webs. Nature (London) 409:78-81.

Paine, R. T. 1988. Food webs: Road maps of interactions or grist for theoretical development? Ecology 69:1648-1654

Paine, R. T. 1992. Food-web analysis through field measurement of per capita interaction strength. Nature (London) 355:73-75.

Pielou, E. C. 1966a. The measurement of diversity in different types of biological collections. Journal of Theoretical Biology 13:131-144.

Pielou, E. C. 1966b. Shannon's formula as a measure of specific diversity: its use and misuse. American Naturalist 100:463-465.

Pimm, S. L. 1982. Food webs. Chapman and Hall, London, UK.
Pimm, S. L. 1984. The complexity and stability of ecosystems. Nature (London) 307:321-326.

Pimm, S. L., J. H. Lawton, and J. E. Cohen. 1991. Food web patterns and their consequences. Nature (London) 350: 669-674.

Polis, G. A. 1991. Complex trophic interactions in deserts: an empirical critique of food-web theory. American Naturalist 138: 123-155.

Polis, G. A. 1994. Food-web, trophic cascades and community structure. Australian Journal of Ecology 19:121-136.

Polis, G. A., and S. D. Hurd. 1996. Linking marine and terrestrial food webs: allochthonous input from the ocean supports high secondary produtivity on small islands and coastal land communities. American Naturalist 147:396-423.

Polis, G. A., and D. R. Strong. 1996. Food web complexity and community dynamics. American Naturalist 147:813-846.

Raffaelli, D. G., and S. J. Hall. 1996. Assessing the relative importance of trophic links in food webs. Pages 185-191 in G. A. Polis, and K. O. Winemiller, editors. Food webs: integration of patterns and dynamics. Chapman and Hall, New York, New York, USA.

Schoener, T. W. 1989. Food webs from the small to the large. Ecology 70:1559-1589.

Shannon, C. E. 1948. A mathematical theory of communications. Bell System Technical Journal 27:379-423.

Simpson, E. H. 1949. Measurement of diversity. Nature 163: 688.

Smith, B., and J. B. Wilson. 1996. A consumers guide to evenness indices. Oikos 76:70-82.

Solow, A. R., and A. R. Beet. 1998. On lumping species in food webs. Ecology 79:2013-2018.

Sprules, W. G., and J. E. Bowerman. 1988. Omnivory and food chain length in zooplankton food webs. Ecology 69: 418-426.

Sugihara, G. 1982. Niche hierarchy: structure assembly and organization in natural communities. Dissertation. Princeton University, Princeton, New Jersey, USA.

Sugihara, G. 1984. Graph theory, homology, and food webs. Pages 83-101 in S. A. Levin, editor. Population biology. Proceedings of Symposia in Applied Mathematics. Volume 30. American Mathematical Society, Providence, Rhode Island, USA.

Sugihara, G., K. Schoenly, and A. Trombla. 1989. Scale invariance in food web properties. Science 245:48-52.

Tavares-Cromar, A. F., and D. D. Williams. 1996. The importance of temporal resolution in food web analysis: Evidence for a detritus-based stream. Ecological Monographs 66:91-113.

Ulanowicz, R. E. 1986. Growth and development: ecosystems phenomenology. Springer Verlag, New York, New York, USA.

Ulanowicz, R. E. 1997. Ecology, the ascendent perspective. Columbia University Press, New York, New York, USA

Ulanowicz, R. E., and W. F. Wolff. 1991. Ecosystem flow networks: Loaded dice? Mathematical Biosciences 103:45-68.

Warren, P. H. 1989. Spatial and temporal variation in the structure of a freshwater food web. Oikos 55:299-311.

Warren, P. H. 1994. Making connections in food webs. Trends in Ecology and Evolution 9:136-141.

Williams, R. J., and N. D. Martinez. 2000. Simple rules yield complex food webs. Nature (London) 404:180-183.

Winemiller, K. O. 1990. Spatial and temporal variation in tropical fish trophic networks. Ecological Monographs 60: 331-367.

Wootton, J. T. 1994. Predicting direct and indirect effects: an integrated approach using experiments and path analysis. Ecology 75:151-165.

Yodzis, P. 1989. Introduction to theoretical ecology. Harper and Row, New York, New York, USA. 


\section{APPENDIX}

Example of a quantitative food-web matrix, representing the annual carbon flows among the 33 major taxa of the Chesapeake Bay mesohaline ecosystem (data from Baird and Ulanowicz 1989).

\begin{tabular}{cccccccccccccc}
\hline \hline Taxon & 6 & 7 & 8 & 9 & 10 & 11 & 12 & 13 & 14 & 15 & 16 & 17 \\
\hline 1 & 0 & 31715 & 37149 & 0 & 0 & 4199 & 2275 & 4415 & 0 & 0 & 0 & 0 \\
2 & 0 & 870.9 & 1685.4 & 131.5 & 0 & 105.2 & 56.9 & 110.6 & 0 & 0 & 0 & 0 \\
3 & 0 & 0 & 0 & 0 & 0 & 0 & 0 & 0 & 160831 & 25062.2 & 57334.5 & 35961.7 \\
4 & 0 & 0 & 0 & 0 & 0 & 0 & 0 & 0 & 0 & 0 & 0 & 18086 \\
5 & 88721 & 0 & 0 & 0 & 0 & 0 & 0 & 0 & 0 & 0 & 0 & 0 \\
6 & 0 & 31638 & 0 & 0 & 0 & 0 & 0 & 0 & 0 & 0 & 0 & 0 \\
7 & 0 & 0 & 7555 & 3437 & 0 & 290 & 156 & 304 & 0 & 0 & 0 & 0 \\
8 & 0 & 0 & 0 & 6878 & 0 & 0 & 0 & 0 & 0 & 0 & 0 & 0 \\
9 & 0 & 0 & 0 & 0 & 1159 & 0 & 0 & 0 & 0 & 0 & 0 & 0 \\
11 & 0 & 0 & 0 & 0 & 552 & 0 & 0 & 0 & 0 & 0 & 0 & 0 \\
12 & 0 & 0 & 0 & 0 & 0 & 0 & 0 & 0 & 0 & 0 & 0 & 0 \\
14 & 0 & 0 & 0 & 0 & 0 & 0 & 0 & 0 & 0 & 0 & 0 & 0 \\
15 & 0 & 0 & 0 & 0 & 0 & 0 & 0 & 0 & 0 & 0 & 0 & 0 \\
16 & 0 & 0 & 0 & 0 & 0 & 0 & 0 & 0 & 0 & 0 & 0 & 0 \\
18 & 0 & 0 & 0 & 0 & 0 & 0 & 0 & 0 & 0 & 0 & 0 & 0 \\
19 & 0 & 0 & 0 & 0 & 0 & 0 & 0 & 0 & 0 & 0 & 0 & 0 \\
21 & 0 & 0 & 0 & 0 & 0 & 0 & 0 & 0 & 0 & 0 & 0 & 0 \\
22 & 0 & 0 & 0 & 0 & 0 & 0 & 0 & 0 & 0 & 0 & 0 & 0 \\
23 & 0 & 0 & 0 & 0 & 0 & 0 & 0 & 0 & 0 & 0 & 0 & 0 \\
27 & 0 & 0 & 0 & 0 & 0 & 0 & 0 & 0 & 0 & 0 & 0 \\
31 & 0 & 0 & 0 & 0 & 0 & 0 & 0 & 0 & 0 & 0 & 0 \\
\hline
\end{tabular}

Notes: Flows are in milligrams of $\mathrm{C}$ per square meter per year. Rows are consumed taxa, and columns consumer taxa. Only living compartments are included in this version of the food web; ignored compartments are: exogenous inputs, dissolved organic carbon, suspended particulate organic carbon, sediment organic carbon, exploitation, exports, and respiration. Columns and rows with zero marginal sums are excluded for economy. Taxa key: $1=$ phytoplankton; 2 = bacteria attached to suspended particles; $3=$ sediment bacteria; $4=$ benthic algae; $5=$ free bacteria in water column; $6=$ heterotrophic microflagellates; $7=$ microzooplankton; $8=$ zooplankton; $9=$ ctenophores; $10=$ sea nettles, Chrysaora quinquecirrha; $11=$ other suspension feeders; $12=$ Mya; 13 = oysters, Crassostrea virginica; $14=$ other polychaetes; $15=$ Nereis; $16=$ Macoma spp.; $17=$ meiofauna; $18=$ crustacean deposit feeders; $19=$ blue crabs Callinectes sapidus; $20=$ fish larvae; $21=$ alewife Alosa pseudoharengus and herring (Clupeidae Fam.); 22 = bay anchovy Anchoa mitchilli; 23 = menhaden Brevoortia tyrannus; $24=$ shad Alosa sapidissima; $25=$ atlantic croaker Micropogonius undulatus; $26=$ hog choker Trinectes maculatus; $27=$ spot Leiostomus xanthurus; 28 = white perch Morone americana; 29 = catfish Arius felis; 30 = blue fish Pomatomus saltatrix; 31 = weak fish Cynoscion aregalis; $32=$ summer flounder Paralichthys dentatus; $33=$ striped bass Morone saxatilis. 
APPENDIX. Extended.

\begin{tabular}{ccccccccccccccccc}
\hline \hline 18 & 19 & 20 & 21 & 22 & 23 & 24 & 25 & 26 & 27 & 28 & 29 & 30 & 31 & 32 & 33 \\
\hline 0 & 0 & 0 & 0 & 277 & 20.8 & 0 & 0 & 0 & 0 & 0 & 0 & 0 & 0 & 0 & 0 \\
0 & 0 & 0 & 0 & 12.3 & 4.2 & 0 & 0 & 0 & 0 & 0 & 0 & 0 & 0 & 0 & 0 \\
14075.2 & 1690.3 & 0 & 0 & 0 & 0 & 0 & 0 & 0 & 0 & 0 & 0 & 0 & 0 & 0 & 0 \\
0 & 0 & 0 & 0 & 0 & 0 & 0 & 0 & 0 & 0 & 0 & 0 & 0 & 0 & 0 & 0 \\
0 & 0 & 0 & 0 & 0 & 0 & 0 & 0 & 0 & 0 & 0 & 0 & 0 & 0 & 0 & 0 \\
0 & 0 & 0 & 0 & 0 & 0 & 0 & 0 & 0 & 0 & 0 & 0 & 0 & 0 & 0 & 0 \\
0 & 0 & 0 & 0 & 0 & 0 & 0 & 0 & 0 & 0 & 0 & 0 & 0 & 0 & 0 & 0 \\
0 & 0 & 4.9 & 25.7 & 1534 & 248.2 & 5.2 & 0 & 0 & 0 & 0 & 0 & 0 & 0 & 0 & 0 \\
0 & 0 & 0 & 0 & 0 & 0 & 0 & 0 & 0 & 0 & 0 & 0 & 0 & 0 & 0 & 0 \\
0 & 538 & 0 & 0 & 0 & 0 & 0 & 0 & 0 & 0 & 0 & 0 & 0 & 0 & 0 & 0 \\
0 & 215 & 0 & 0 & 0 & 0 & 0 & 0 & 9 & 0 & 0 & 0 & 0 & 0 & 0 & 0 \\
0 & 0 & 0 & 0 & 0 & 0 & 0 & 7.2 & 59 & 314 & 76 & 152.8 & 0 & 0 & 0 & 0 \\
0 & 352 & 0 & 0 & 0 & 0 & 0 & 2.1 & 14 & 97 & 59 & 22.6 & 0 & 0 & 0 & 0 \\
0 & 4538 & 0 & 0 & 0 & 0 & 0 & 0 & 0 & 55 & 0 & 0 & 0 & 0 & 0 & 0 \\
0 & 967 & 0 & 0 & 0 & 0 & 0 & 0.3 & 14 & 2 & 0 & 43.1 & 0 & 0 & 0.9 & 0 \\
0 & 293 & 0 & 0 & 0 & 0 & 0 & 0 & 0 & 0 & 0 & 0 & 0 & 0 & 0 & 2.4 \\
0 & 0 & 0 & 0 & 0 & 0 & 0 & 0 & 0 & 0 & 0 & 0 & 0 & 0 & 0 & 0.2 \\
0 & 0 & 0 & 0 & 0 & 0 & 0 & 0 & 0 & 14.5 & 14.6 & 0 & 2.7 & 91.4 & 12.3 & 17.2 \\
0 & 0 & 0 & 0 & 0 & 0 & 0 & 0 & 0 & 0 & 0 & 0 & 2.6 & 0 & 8 & 10.6 \\
0 & 0 & 0 & 0 & 0 & 0 & 0 & 0 & 0 & 0 & 0 & 0 & 10.2 & 0 & 0 & 0 \\
0 & 0 & 0 & 0 & 0 & 0 & 0 & 0 & 0 & 0 & 0 & 0 & 0 & 0 & 4.3 & 0 \\
\hline
\end{tabular}

